\title{
Friedel-Crafts acylation of 2-methoxynaphthalene over zeolite catalysts
}

\author{
Debasish Das, Soofin Cheng* \\ Chemistry Department, National Taiwan University, 1 Roosevelt Road, Section 4, Taipei 106, Taiwan
}

Received 17 May 1999; received in revised form 10 December 1999; accepted 15 December 1999

\begin{abstract}
The Friedel-Crafts acylation of 2-methoxynaphthalene was carried out in the liquid-phase batch conditions using Hmordenite, H-beta and H-Y zeolite as catalysts. All the catalysts showed 35-40\% conversion in the temperature range of $100-150^{\circ} \mathrm{C}$. 1-Acyl-2-methoxynaphthalene was formed as the primary product. When acetyl chloride was used as the acylating agent, a higher yield of 6-acyl-2-methoxynaphthalene was obtained through rearrangement of the sterically hindered 1-acyl isomer to the 6-acyl isomer. The presence of extra-framework aluminum in the catalyst facilitates the isomerization of 1-acyl isomer to the desired 6-acyl isomer. The final product selectivity was found to be more dependent on the nature of the acylating agents and the reaction temperature and less on the type of the zeolite structure. (C) 2000 Elsevier Science B.V. All rights reserved.
\end{abstract}

Keywords: Friedel-Crafts acylation; Zeolites; H-mordenite; H-beta; H-Y; 2-Methoxynaphthalene

\section{Introduction}

Friedel-Crafts acylation is one of the most important methods for the synthesis of aromatic ketones [1]. The synthesis of aromatic ketones is of special importance in the fine chemicals industry, as many synthetic fragrances and pharmaceuticals contain an acyl group. In general, Lewis-acid metal chloride catalysts are employed for aromatic acylation. Unfortunately, use of such conventional catalysts is associated with a number of problems. A major drawback of these catalysts is that they are non-regenerable and more than a stoichiometric amount of the catalyst is needed. Besides, the work-up steps involving hydrolysis of the resultant intermediate complex produce hazardous

\footnotetext{
* Corresponding author. Tel.: +886-2-2363-8017; fax: $+886-2-2363-6359$.
}

corrosive waste products. Disposal of such waste products also leads to other environmental problems.

Many of these drawbacks can be overcome by using recoverable and regenerable solid catalysts like zeolites. Therefore, the use of zeolites and other solid acid catalysts in the manufacture of fine chemicals and chemical intermediates is gaining much more attention in recent years. The Friedel-Crafts acylation reaction mechanism, as proposed by Olah [1], involves acylation either by an adduct of the catalyst and the acylating agent or by free acylium ions, depending on the reaction conditions. In heterogeneous zeolite-catalysed acylation, a similar mechanism is assumed to apply, in which the adduct is formed by the interaction of the surface acid sites with an acylating agent (Fig. 1). Chiche et al. [2] investigated the acylation of toluene and xylene by a series of straight-chain carboxylic acids over lanthanide-exchanged zeolites. 

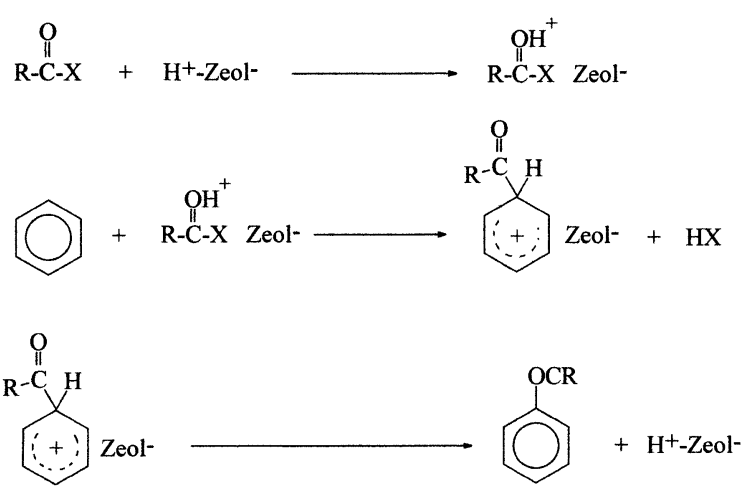

Fig. 1. Mechanism of zeolite catalyzed acylation of aromatics.

Similar reactions over USY and ZSM-5 zeolites were reported recently in the literature $[3,4]$. Acylation of aromatic ethers like anisole [5-8], phenetole [8], veratrole [9] and 2-methoxynaphthalene [9-11] over zeolite catalysts was also reported. Acylation of 2-methoxynaphthalene is of particular interest if the ketone functionality can be placed at the 6-position, since this compound could then be used as a precursor to the anti-inflammatory drug naproxen [12]. Harvey and Mäder [10] studied this reaction over zeolite USY, H-beta and ZSM-12 and showed that in all cases 1-acyl-2-methoxynaphthalene was obtained as the major product. In comparison to other zeolites, H-beta with narrower 12-ring channels and no super cages showed slightly increased selectivity for 6-acyl-2-methoxynaphthalene. It was proposed that the more confined internal environment of $\mathrm{H}$-beta restricts access to the 1-position of 2-methoxynaphthalene [10]. Recently, mesoporous molecular sieve material of MCM-41 type [11] and cation-exchanged clays [9] were also studied for the acylation of 2-methoxynaphthalene. However, in both cases, acylation occurred predominantly in the 1-position only. On the other hand, it was noticed that with Zn-impregnated MCM-41 high selectivity to 6-acyl-2-methoxynaphthalene can be obtained in polar solvents like nitrobenzene [11]. Thus, it is clear that the selectivity to the 6-acyl-2-methoxynaphthalene is not only controlled by the pore geometry or pore structure of the zeolite used, other factors also play important roles.

In the present work, acylation of 2-methoxynaphthalene was carried out in the liquid-phase conditions in the presence of H-mordenite zeolite. Effects of reaction temperature, nature of the acylating agent, and the substrate/acylating agent ratio were also investigated in order to increase the yield of the 6-acyl isomer. Acylation of 2-methoxynaphthalene over other zeolites has been reported earlier [10]. In this work the results obtained with $\mathrm{H}$-mordenite zeolite were compared with those obtained with other zeolites like H-beta and HY. Unlike H-beta and HY, which have three-dimensional channel structures, H-mordenite possesses a two-dimensional channel structure. The effect of the zeolite structure on product selectivity was also examined.

\section{Experimental}

Zeolite mordenite $(\mathrm{Si} / \mathrm{Al}=9.5)$ was received in its protonic form from Tosoh Chemicals as Reference Catalyst No. 28. Zeolite $\mathrm{NH}_{4}$-beta and $\mathrm{NH}_{4}-\mathrm{Y}$ were obtained from PQ Corporation and Advchem laboratories, respectively. They were converted into their protonic forms by calcination in air at $550^{\circ} \mathrm{C}$ for $6 \mathrm{~h}$. The Si/Al ratio, ion-exchange level and the surface area of the zeolite samples are given in Table 1. Acid-washed mordenite sample (to remove extra-framework aluminum) was prepared by stirring the parent sample with dilute hydrochloric acid $(0.5 \mathrm{~N})$ in a beaker for $15 \mathrm{~min}$. The solid was then filtered, washed with water and dried at $120^{\circ} \mathrm{C}$.

Acylation reaction was carried out under batch conditions with reflux set-up in the temperature range of $100-150^{\circ} \mathrm{C}$. Both acetyl chloride and acetic anhydride were used as the acylating agents to study the effect of the nature of the acylating agent. Sulfolane (bp $285^{\circ} \mathrm{C}$ ) was used as the solvent. A typical reaction condition was as follows: $3.2 \mathrm{mmol}$ of 2-methoxynaphthalene and $3.2 \mathrm{mmol}$ of acetyl chloride were well mixed in

Table 1

Physical properties of the zeolite samples

\begin{tabular}{lrlc}
\hline $\begin{array}{l}\text { Zeolite } \\
\text { type }\end{array}$ & Si/Al & $\begin{array}{l}\text { Ion-exchange } \\
\text { level }(\%)\end{array}$ & $\begin{array}{l}\text { Surface } \\
\text { area }\left(\mathrm{m}^{2} / \mathrm{g}\right)\end{array}$ \\
\hline Mordenite & 9.5 & 98 & 380 \\
Beta & 12.5 & 97 & 680 \\
Y & 2.6 & 92 & 560 \\
Mordenite (acid-washed) & 10.7 & - & - \\
\hline
\end{tabular}


$10 \mathrm{ml}$ solvent along with $1 \mathrm{ml}$ nitrobenzene (internal standard). To this mixture $150 \mathrm{mg}$ of freshly activated $\left(400^{\circ} \mathrm{C}, 2 \mathrm{~h}\right)$ catalyst was added quickly while still hot. The reaction temperature was then raised slowly to the desired value. Samples were taken periodically and analyzed by gas chromatography (Restek-1 capillary column, $30 \mathrm{~m}$ ) to monitor the progress of the reaction. Product identification was carried out by using available standard compounds and also by GC-Mass analysis (HP 6890-5973). All the chemicals (AR grade) were obtained from Aldrich or Acros Organics and were used without further purification.

In order to examine the regenerability of the catalyst, the solid phase was separated from the reaction mixture by filtration, washed with dichloromethane, dried at $100^{\circ} \mathrm{C}$ and finally calcined at $500^{\circ} \mathrm{C}$ for $24 \mathrm{~h}$ to remove the carbonaceous deposits before further use.

$\mathrm{X}$-ray powder diffraction patterns were recorded on a Scintag X1 diffractometer using $\mathrm{Cu} \mathrm{K} \alpha$ radiation $(\lambda=0.154 \mathrm{~nm})$. Solid state ${ }^{27} \mathrm{Al}$ MAS NMR measurements were carried out using a Bruker MSL 500 spectrometer with $4 \mathrm{~mm}$ cylindrical zirconia rotors spun at $12 \mathrm{kHz}$. Spectra were obtained at $130.319 \mathrm{MHz}$ with $20^{\circ}$ pulse duration of $1 \mu \mathrm{s}$ and recycle delay of $200 \mathrm{~ms}$. Chemical shifts were recorded with reference to aqueous $\mathrm{Al}\left(\mathrm{NO}_{3}\right)_{3}$ solution.

\section{Results and discussion}

Fig. 2 shows the change in 2-methoxynaphthalene conversion with reaction time at different reaction temperatures and also using different acylating agents. Acetyl chloride and acetic anhydride both can create only one acyl group. Acetic acid formed in the reaction with acetic anhydride is not reactive, as separate experiments conducted with acetic acid showed that it has no acylation activity under the reaction conditions. It was found that at $100^{\circ} \mathrm{C}$ acetyl chloride showed much higher conversion than that obtained with acetic anhydride. It appears that acetyl chloride is more effective for acylation than acetic anhydride. As the reaction temperature was raised, conversion increased rapidly and the maximum value was reached much faster. At higher temperatures, both acylating agents yielded very similar conversions. The conversion reached the maximum value of ca. $30 \%$ at around $9 \mathrm{~h}$ TOS at $125^{\circ} \mathrm{C}$. However, further increase in reaction

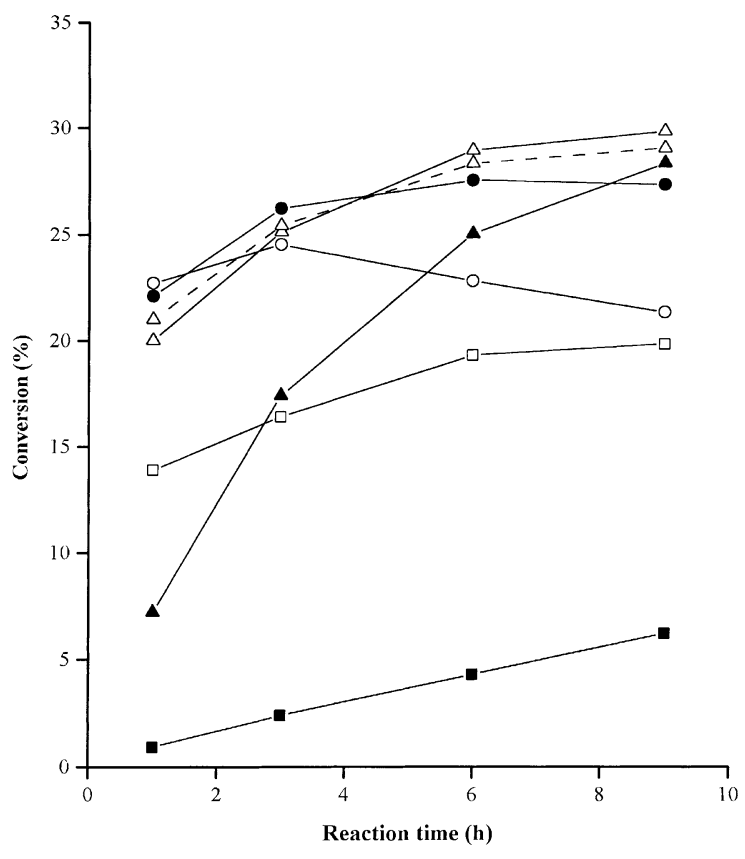

Fig. 2. Conversion of 2-methoxynaphthalene as a function of reaction time on $\mathrm{H}$-mordenite catalysts using acetyl chloride (open symbols) and acetic anhydride (solid symbols) (substrate:acylating agent $=1: 1$ molar ratio); $\left(\square, \mathbf{\square}: 100^{\circ} \mathrm{C} ; \Delta, \boldsymbol{\Delta}: 125^{\circ} \mathrm{C}, \bigcirc\right.$, $150^{\circ} \mathrm{C}$; dotted lines for conversion with acid-washed mordenite at $125^{\circ} \mathrm{C}$ ).

temperature reduced the conversion by facilitating the reverse deacylation reaction [10]. Such decrease in conversion by deacylation was more noticeable at higher reaction temperatures.

Higher conversion with acid chloride over acid anhydride was also observed in the benzoylation of $m$-xylene [3]. The authors proposed that the acid chloride or $\mathrm{HCl}$ liberated during the reaction interacted with the extra-framework aluminum species in the catalyst to form catalytically active aluminum complexes. However, we have not observed any significant difference in conversion (Fig. 2) when acid-washed mordenite (to remove extra-framework aluminum) was used as catalyst.

The distribution of the three acylated isomers obtained with both acetyl chloride and acetic anhydride at $125^{\circ} \mathrm{C}$ are given in Fig. 3. It can be seen that, with acetic anhydride, 1-acyl-2-methoxynaphthalene was obtained as the major product, as observed earlier by other workers also [9-11]. As the reaction proceeded, a 


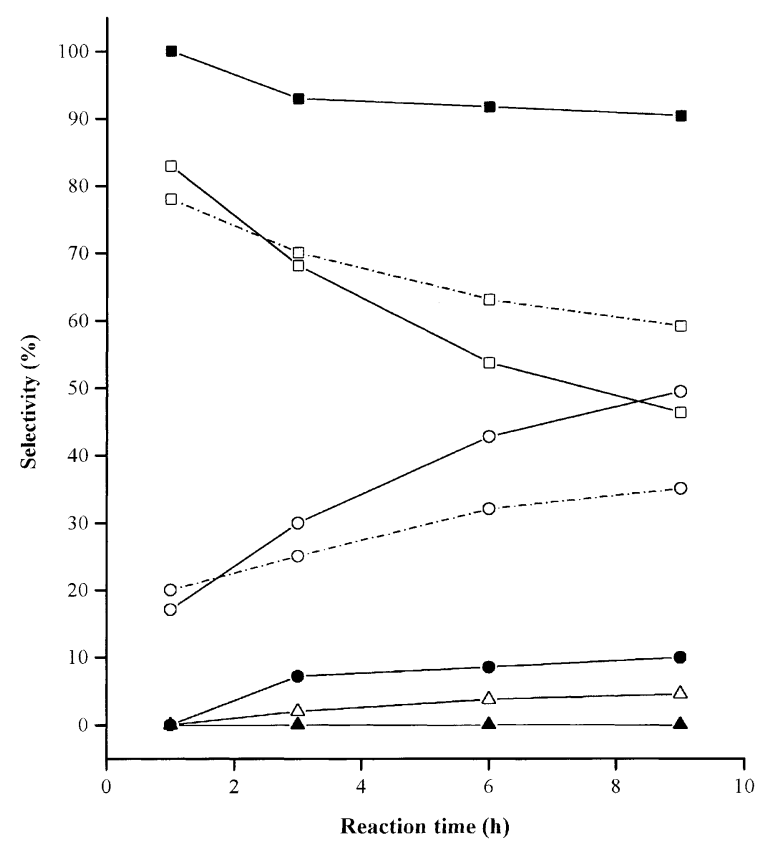

Fig. 3. Selectivity to different acyl-2-methoxynaphthalenes as a function of reaction time on $\mathrm{H}$-mordenite catalysts at $125^{\circ} \mathrm{C}$ using acetyl chloride (open symbols) and acetic anhydride (solid symbols) (substrate:acylating agent $=1: 1$ molar ratio); ( $\square, \mathbf{\square}$ : 1-acyl-2-methoxynaphthalene, $\triangle, \boldsymbol{\Delta}$ : 8-acyl-methoxynaphthalene, O, 6-acyl-2-methoxynaphthalene; dotted lines for selectivity with acid-washed mordenite catalyst).

minor quantity $(<10 \%)$ of 6-acyl-2-methoxynaphthalene was formed and a negligible amount of 8acyl-2-methoxynaphthalene was detected. However, the product pattern obtained with acetyl chloride as the acylating agent was quite different. Initially, 1-acyl-2-methoxynaphthalene was the predominant product. Nevertheless, as the reaction proceeded, its selectivity decreased markedly, accompanied by an increase in 6-acyl-2-methoxynaphthalene selectivity as well as by formation of a small quantity $(<5 \%)$ of 8-acyl-2-methoxynaphthalene. The sharp decrease of the 1-acyl isomer with concomitant increase of the other two isomers suggests that the 6- and 8-acyl isomers probably arise from the isomerization of the primary product, 1-acyl-2-methoxynaphthalene.

It is well known that the presence of an electrondonating group like methoxy activates the 1-, 6, and 8-positions of the naphthalene ring. The 1-position is more active than the other two positions, and acy-

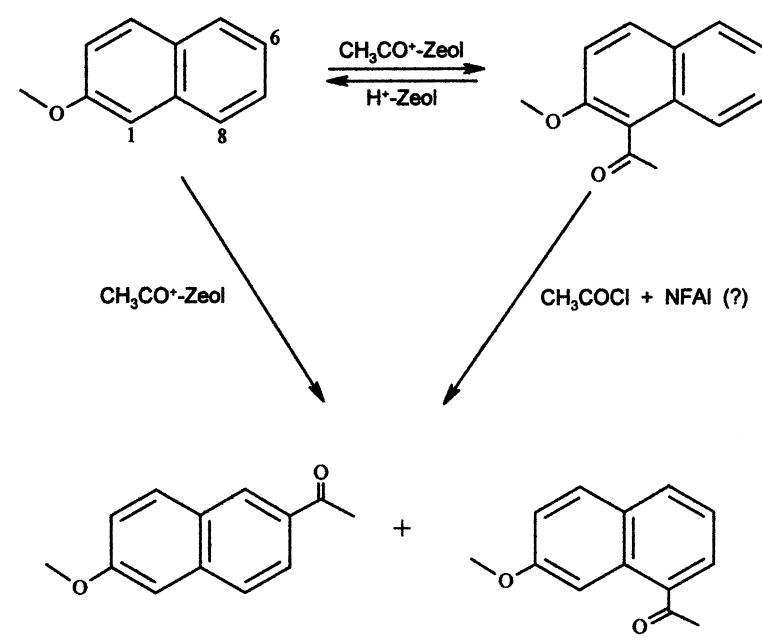

Scheme 1. Acylation of 2-methoxynaphthalene and isomerization of the acyl group.

lation of 2-methoxynaphthalene generally occurs at this kinetically controlled position. However, migration of the acyl group from 1- to 6-position [13] and protiodeacylation of the acyl group at the 1-position [14] results in the formation of the thermodynamically most stable 6-acylated isomer (Scheme 1). Steric hindrance to acylation is in the order 1- >8$>6$-position. Hence, the isomerisation of the sterically hindered ketones like 1-acyl-2-methoxynaphthalene to sterically less hindered isomers like 6- and 8-acyl-2-methoxynaphthalene will be favored.

Fig. 4 shows the plots of yields of individual isomers with conversion of 2-methoxynaphthalene using both acetic anhydride and acetyl chloride. It can be seen that, with acetic anhydride, yields of 1- and 6-acyl-2-methoxynaphthalene both increased linearly with the rise in conversion. However, yields of 1-acyl-2-methoxynaphthalene were much higher than those for 6-acyl-2-methoxynaphthalene. On the other hand, with acetyl chloride as acylating agent, yields of 1-acyl-2-methoxynaphthalene reached a maximum at about $25 \%$ conversion and decreased with further increase in conversion, whereas yields of 6-acyl-2-methoxynaphthalene were much lower initially but increased continuously with increase in conversion. It appears that the reaction follows two different mechanisms [15] with acetic anhydride and acetyl chloride. In the former case, formation of the 


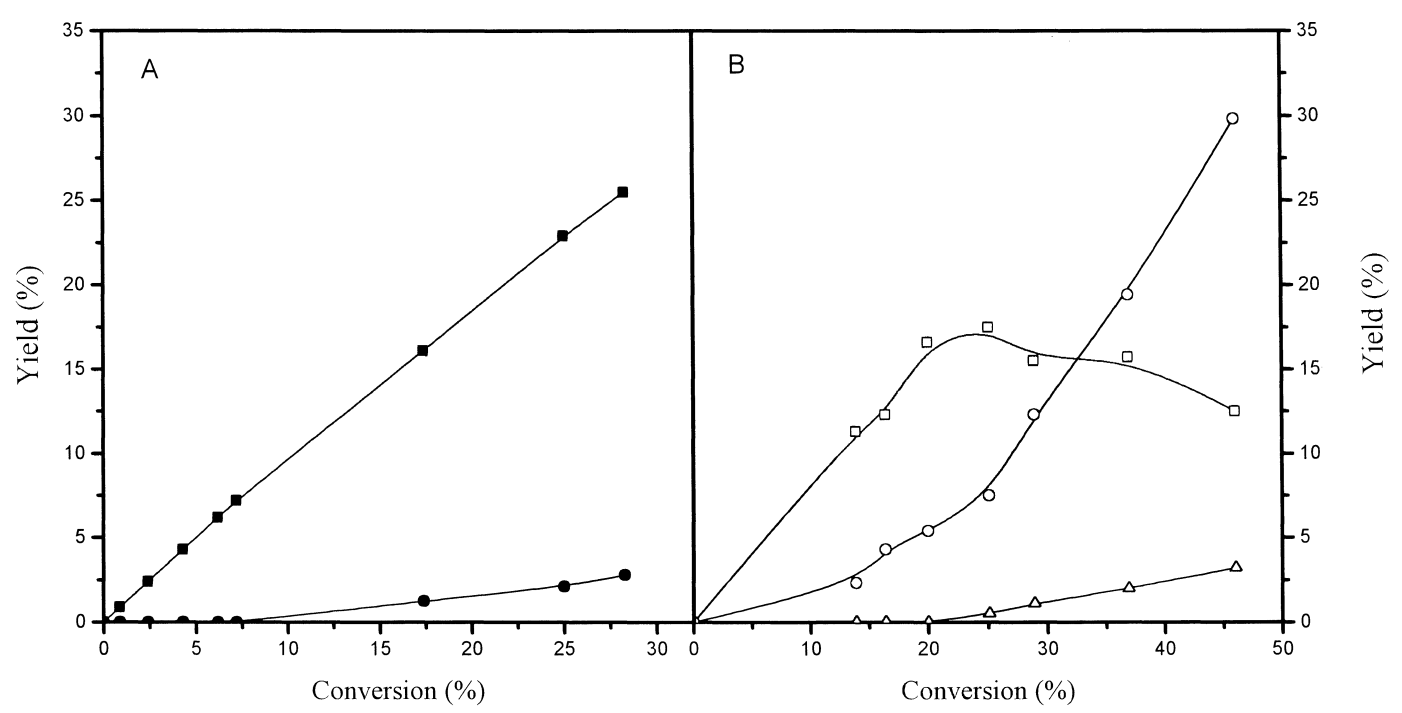

Fig. 4. Yields of different acyl-2-methoxynaphthalenes as a function of conversion with H-mordenite catalysts at $125^{\circ} \mathrm{C}$ using (A) acetic anhydride and (B) acetyl chloride (substrate:acylating agent=1:1 molar ratio); ( $\square$, $\mathbf{\square}$ : 1-acyl-2-methoxynaphthalene, $\Delta, \mathbf{\Delta}$ 8-acyl-2-methoxynaphthalene, $\bigcirc$, О: 6-acyl-2-methoxynaphthalene).

1-acyl and 6-acyl isomers are independent of each other and possibly formed via two parallel reactions. Conversion of 1-acyl to other stable isomers like 6-acyl and 8-acyl was negligible.

In the latter case, the reaction follows a combination of parallel and sequential paths. Initially, at lower conversion, 1-acyl-2-methoxynaphthalene was the major product, but at higher conversions, it isomerizes to more stable 6-acyl-2-methoxynaphthalene. Isomerization of 1-acyl isomer was found to be enhanced by the presence of extra-framework aluminum species (vide infra).

2-Methoxynaphthalene $+\left(\mathrm{CH}_{3} \mathrm{CO}\right)_{2} \mathrm{O}$

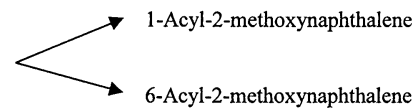

2-Methoxynaphthalene $+\mathrm{CH}_{3} \mathrm{COCl}$

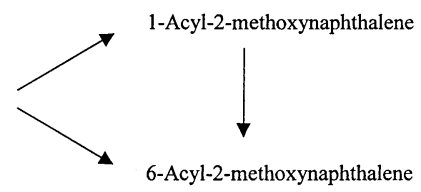

Fig. 5 shows the variation in the yields of different acylated isomers with reaction time at $125^{\circ} \mathrm{C}$. It can be seen that the yields of 6-acyl-2-methoxynaphthalene increased much more rapidly when acetyl chloride was used as the acylating agent. Conversely, yields of 6-acyl-2-methoxynaphthalene remained almost constant at $<2 \%$ with acetic anhydride.

These results show that, with acetyl chloride as the acylating agent, acylation and consecutive rearrange-

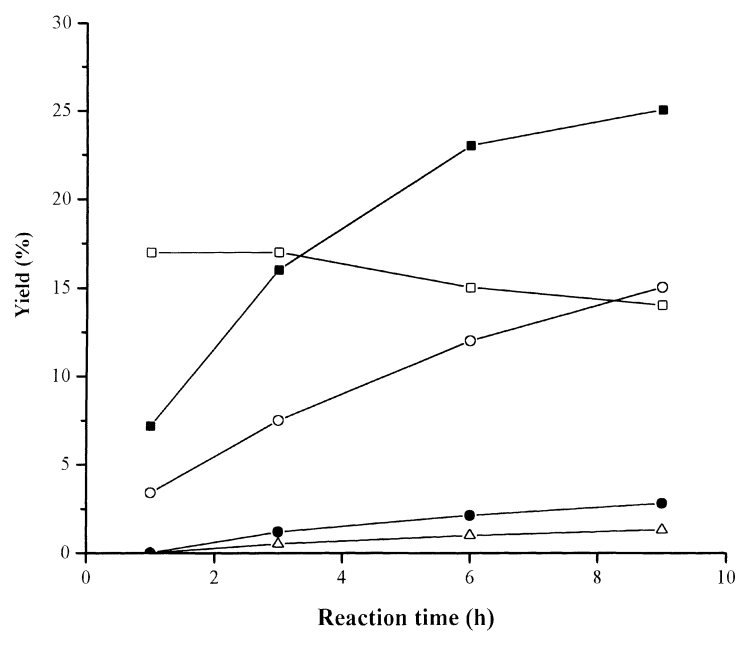

Fig. 5. Yields of different acyl-2-methoxynaphthalenes as a function of reaction time on $\mathrm{H}$-mordenite catalysts at $125^{\circ} \mathrm{C}$ using acetyl chloride (open symbols) and acetic anhydride (solid symbols) (substrate: acylating agent $=1: 1$ molar ratio); ( $\square$, $\mathbf{\square}$ : 1-acyl-2-methoxynaphthalene, $\Delta, \boldsymbol{\Delta}$ : 8-acyl-2-methoxynaphthalene, $\bigcirc$, : 6-acyl-2-methoxynaphthalene). 
ment of the 1-acyl isomer leads to the increased formation of the desired 6-acyl isomer. In contrast, with acetic anhydride, the rearrangement of the 1-acyl isomer was very limited and the product mostly contains only 1 -acyl isomer and very little 6-acyl isomer. The maximum yield of 6-acyl-2-methoxynaphthalene that can be achieved by changing the experimental parameters like temperature, reaction time, substrate/acylating ratio was found to be only $15 \%$ with acetic anhydride.

It appears that the rearrangement of 1-acyl isomer (through deacylation-reacylation) is more favored in the presence of acetyl chloride than in that of acetic anhydride. Deacylation may also be facilitated by the $\mathrm{HCl}$ liberated from acetyl chloride during the course of the reaction. However, when $0.5 \mathrm{ml}$ of $37 \mathrm{wt} . \%$
$\mathrm{HCl}$ acid was added to a reaction mixture containing 2-methoxynaphthalene and acetic anhydride, after $1 \mathrm{~h}$ of reaction at $150^{\circ} \mathrm{C}$ no sharp change in the product selectivity was observed. After further $9 \mathrm{~h}$ of reaction the product mostly contained only 1 -acyl isomer. This indicates that rearrangement of the 1-acyl isomer is not caused by $\mathrm{HCl}$ liberated from acetyl chloride. It was reported earlier that extra-framework aluminum species present in the catalyst may react with acetyl chloride to form strong acid centers [3]. Fig. 6 shows that the aluminum atoms in the original H-mordenite sample are mostly in the tetrahedral position. However, a small fraction of octahedral or extra-framework aluminum is also present, as indicated by a broad peak at about 0 ppm. For acid-washed

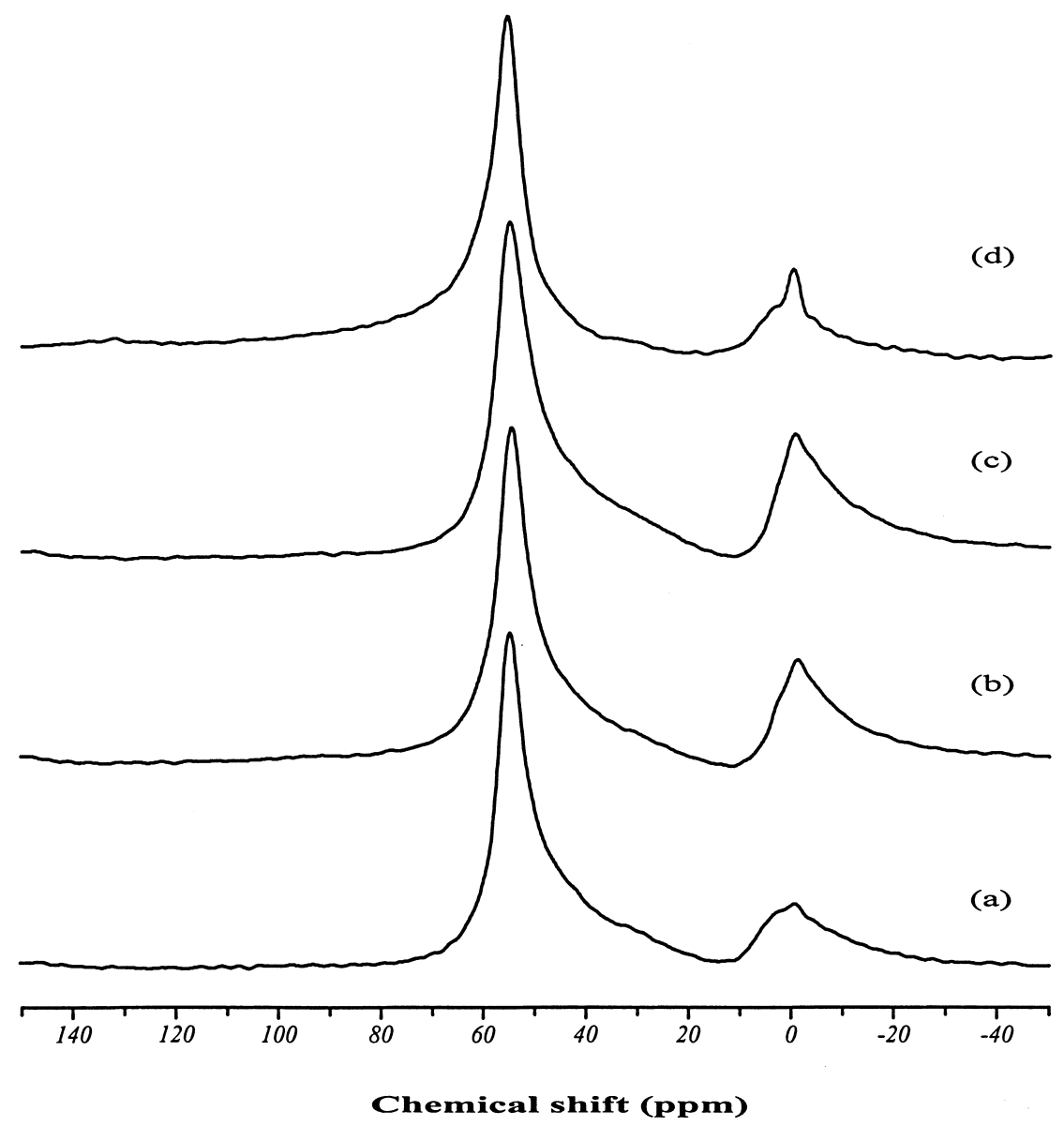

Fig. 6. ${ }^{27} \mathrm{Al}$ MAS NMR spectra of H-mordenite catalyst, (a) fresh, (b) after reaction using acetic anhydride, (c) after reaction using acetyl chloride and (d) acid-washed mordenite. 
mordenite (curve d), the intensity of the peak at $0 \mathrm{ppm}$ was slightly lower, indicating the presence of lower amounts of extra-framework aluminum species. When acid-washed mordenite was used as catalyst, the selectivity towards 6-acyl-isomer (Fig. 3 dotted lines) was found to be less than that obtained with untreated mordenite. This indicates that acetyl chloride may interact with the extra-framework aluminum sites to form strong acid centers which may be responsible for the rearrangement of 1-acyl isomer to the other thermodynamically favored isomers. Earlier workers also noticed that, in the presence of Lewis acid catalysts like Zn-MCM-41, acylation of 2-methoxynaphthalene with acetyl chloride initially formed the sterically hindered 1-acyl-2-methoxynaphthalene as the primary product. However, during the course of the reaction, the 1-acyl isomer easily underwent rearrangement to give higher yields of 6-acyl-2-methoxynaphthalene [11]. Thus, the rearrangement of the primary product, 1-acyl isomer, was most probably caused by the strong acid centers formed by interaction of the extra-framework aluminum species with acetyl chloride.

The effect of reaction temperature on the product distribution is shown in Fig. 7. As observed earlier, selectivity to 6-acyl-2-methoxynaphthalene was always found to be higher with acetyl chloride, whereas acetic anhydride yielded 1-acyl-2-methoxynaphthalene as the major product. Increase in reaction temperature was found to favor the migration of acyl group from 1-position to 6-position. Also, the rearrangement of the 1 -acyl isomer to 6 -and 8 -acyl-isomer was more noticeable when acetyl chloride was used as the acylating agent. With acetyl chloride, the 6-acyl isomer can be obtained with ca. $90 \%$ selectivity at $150^{\circ} \mathrm{C}$.

Increasing the amount of acylating agent in the reaction mixture was found to increase the 2-methoxynaphthalene conversion. Increase of substrate/acylating agent ratio from 1:1 to $1: 2$ results in about $50 \%$ increase in conversion, however, the selectivity towards different acylated isomers did not change much.

For comparison, acylation of 2-methoxynaphthalene with acetyl chloride was also carried out in presence of other zeolites like H-beta and HY; the results are presented in Table 2 . All the three catalysts investigated showed about $30-40 \%$ conversion of 2-methoxynaphthalene in the reaction temperature

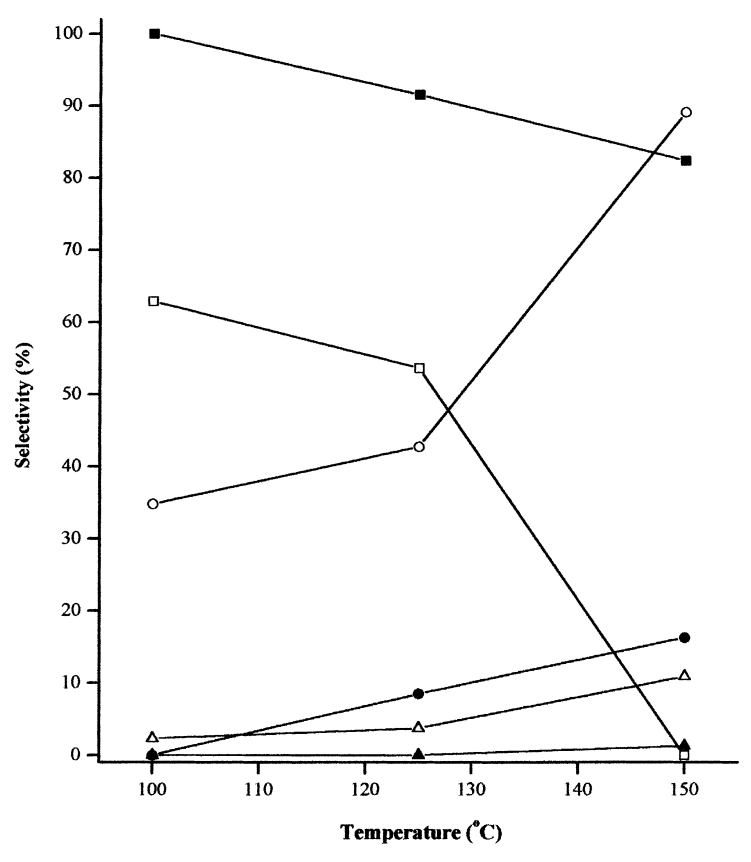

Fig. 7. Selectivity to different acyl-2-methoxynaphthalene isomers as a function of reaction temperature after $3 \mathrm{~h}$ using acetyl chloride (open symbols)and acetic anhydride (solid symbols) (substrate:acylating agent $=1: 1$ molar ratio); ( $\square, \square$ : 1-acyl-2-methoxynaphthalene, $\triangle, \boldsymbol{\Delta}$ : 8-acyl-methoxynaphthalene, , : 6-acyl-2-methoxynaphthalene).

range of $100-150^{\circ} \mathrm{C}$. It was observed that at $100^{\circ} \mathrm{C}$, HY showed higher activity than the other two catalysts. However, as the reaction temperature was raised to $150^{\circ} \mathrm{C}$, the initial high conversion dropped rapidly with the progress of reaction, indicating faster deacylation of the primary product. Zeolite H-beta and $\mathrm{H}$-mordenite also showed some deacylation at higher temperatures, but the drop in conversion was not as sharp as that observed with HY.

Table 2 also shows the selectivity towards different acylated isomers of 2-methoxynaphthalene for different catalysts studied. A high selectivity towards 6-acylated isomer was noticed, especially at a reaction temperature of $150^{\circ} \mathrm{C}$. In the case of HY, larger amounts of 8-acyl isomer were also formed. In contrast, earlier works on the acylation of 2-methoxynaphthalene with acetic anhydride over USY and H-beta showed predominant formation of 1-acyl isomer only [10]. Acylation of 2-methoxynaphthalene on mesoporous molecular 
Table 2

Acylation of 2-methoxynaphthalene by acetyl chloride in sulfolane catalysed by different zeolites ${ }^{\mathrm{a}}$

\begin{tabular}{|c|c|c|c|c|c|}
\hline \multirow[t]{2}{*}{ Catalyst } & \multirow[t]{2}{*}{ Reaction temp. $\left({ }^{\circ} \mathrm{C}\right)$} & \multirow[t]{2}{*}{ Conversion $(\%)^{\mathrm{b}}$} & \multicolumn{3}{|c|}{ Selectivity to different isomers $(\%)^{\mathrm{b}}$} \\
\hline & & & 1-acyl & 6-acyl & 8-acyl \\
\hline \multirow[t]{2}{*}{ H-beta } & 100 & 35 & 30 & 65 & 5 \\
\hline & 150 & 40 & 13 & 77 & 10 \\
\hline \multirow[t]{2}{*}{$\mathrm{H}-\mathrm{Y}$} & 100 & 41 & 60 & 36 & 4 \\
\hline & 150 & 35 & - & 82 & 18 \\
\hline \multirow[t]{2}{*}{ H-mordenite } & 100 & 31 & 59 & 38 & 3 \\
\hline & 150 & 40 & 23 & 69 & 8 \\
\hline
\end{tabular}

${ }^{a}$ Substrate:acylating agent $=1: 2$.

${ }^{\mathrm{b}}$ After $6 \mathrm{~h}$ reaction time.

sieve material, MCM-41 and on cation-exchanged clays was also reported to give substitution predominantly on 1-position only $[9,11]$ as the 1-position in the naphthalene ring is highly activated by the presence of a nearby methoxy group. At the onset of reaction, 1-acyl isomer was formed predominantly but with the progress of reaction most of it rearranged (rearrangement was much faster at higher temperatures) to more stable 6-acyl and 8-acyl isomers. It was observed that at $100^{\circ} \mathrm{C}$ H-beta showed highest selectivity towards 6-acylated isomer (ca. 65\%), while $\mathrm{HY}$ and $\mathrm{H}$-mordenite showed higher selectivity (ca. 60\%) towards sterically hindered 1-acyl isomer.
However, at $150^{\circ} \mathrm{C}$, larger amounts of 6- and 8-acyl isomers were formed at the expense of the 1-acyl isomer. This further confirms that the increased yield of the 6-acyl isomer at higher reaction temperature was due to the rearrangement of the primary product, 1-acyl-2-methoxynaphthalene. Friedel-Crafts acylation is usually irreversible, but at higher temperatures deacylation of the acyl group at 1-position can occur $[10,14]$. Since this phenomenon does not involve thermodynamically more stable and sterically unhindered 6-acyl or less hindered 8-acyl isomers, a high reaction temperature usually leads to a change in the distribution of the products.

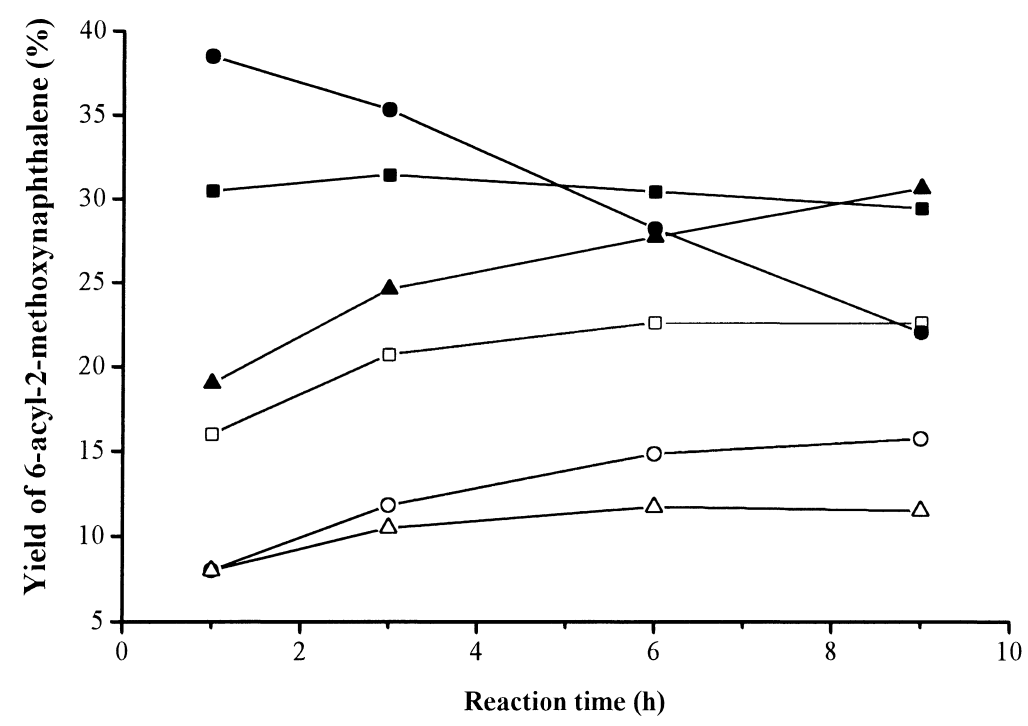

Fig. 8. Yields of 6-acyl-2-methoxynaphthalene with different zeolite catalysts with acetyl chloride as acylating agent (substrate:acylating agent $=1: 2$ molar ratio); (H-beta at $100^{\circ} \mathrm{C} \square, 150^{\circ} \mathrm{C} \mathbf{\square}$ : $\mathrm{HY}$ at $100^{\circ} \mathrm{C} \mathrm{O}, 150^{\circ} \mathrm{C} \boldsymbol{O}$; H-mordenite at $100^{\circ} \mathrm{C} \triangle, 150^{\circ} \mathrm{C} \boldsymbol{\Delta}$ ). 
It appears that at lower reaction temperature, $\mathrm{H}$-beta has better selectivity for 6-acyl isomer than the other two catalysts have. Earlier workers [10] also observed better selectivity for $\mathrm{H}$-beta at $100^{\circ} \mathrm{C}$ and assigned it to the more confined pore geometry in beta zeolite. However, at higher temperatures, rearrangement of 1-acyl isomer to 6-acyl isomer takes place much faster. It can be seen that with HY zeolite 1-acyl isomer has been completely isomerized to other isomers after $6 \mathrm{~h}$ at $150^{\circ} \mathrm{C}$. Isomerization of 1-acyl isomer was relatively less for $\mathrm{H}$-mordenite and $\mathrm{H}$-beta, possibly due to their narrower one-dimensional pore geometry. However, it may be mentioned that the natures of the acylating agent and of the reaction temperature play more important roles than the zeolite structure in determining the final product selectivity.

Fig. 8 shows the yields of 6-acyl-2-methoxynaphthalene with reaction time on different zeolite catalysts. At $100^{\circ} \mathrm{C}, \mathrm{H}$-beta showed much higher yields of 6-acyl isomer than the other two catalysts. Initially H-mordenite and HY showed similar yields. Nevertheless, as the reaction proceeds HY showed higher yields than mordenite. At a higher temperature, e.g., $150^{\circ} \mathrm{C}$, the yields of 6-acyl isomer increased rapidly with mordenite and after $6 \mathrm{~h}$ both mordenite and H-beta showed similar yields. On the other hand, with $\mathrm{HY}$ the yields of 6-acyl isomer decreased continuously at $150^{\circ} \mathrm{C}$ due to decrease in conversion (by deacylation) and also due to increased formation of 8 -acyl isomer. The amount of 8-acyl isomer formed at $150^{\circ} \mathrm{C}$ with $\mathrm{HY}$ was almost twice that produced with H-beta.

Although acetyl chloride showed good conversion and high selectivity towards the 6-acyl-2-methoxynaphthalene, one possible drawback of using acetyl chloride is that the acid liberated during the course of the reaction may affect the crystallinity of the catalyst and also may cause some dealumination. We have checked the crystallinity of the used H-mordenite catalyst by X-ray diffraction and also examined the nature of the aluminum sites by ${ }^{27} \mathrm{Al}$ MAS NMR. Fig. 9 shows the XRD patterns of the parent and the used $\mathrm{H}$-mordenite catalysts. The structure of the zeolite remains intact after the reaction and no appreciable loss of crystallinity was observed. Chemical analysis of the used catalysts showed a slight loss of aluminum after reaction. The ${ }^{27} \mathrm{Al}$ MAS NMR spectra of the parent and used catalysts (Fig. 6) show that after reaction

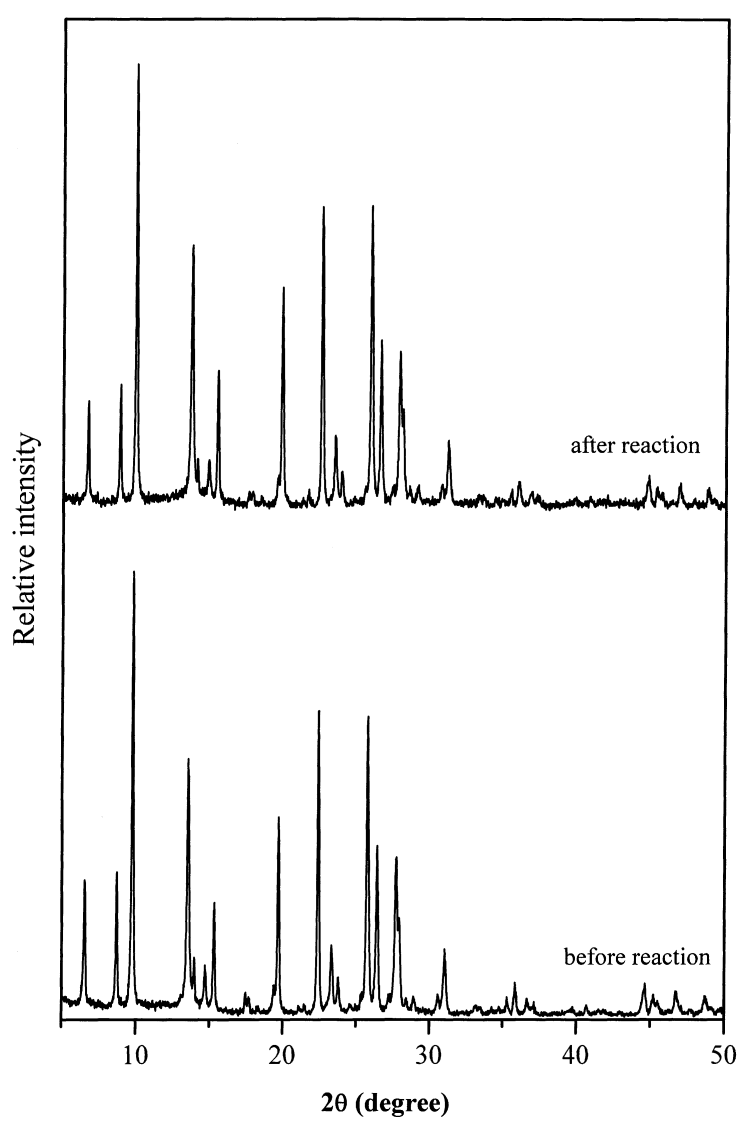

Fig. 9. Powder XRD patterns of the H-mordenite catalyst: (a) before reaction and (b) after reaction.

the intensity of the peak at $0 \mathrm{ppm}$ increased, indicating higher amounts of extra-framework aluminum after reaction. However, both acetyl chloride and acetic anhydride were found to have similar effects.

For practical applications, a good catalyst must be easily regenerable so that it can be used repeatedly.

Table 3

Regenerability of $\mathrm{H}$-mordenite in the acylation of 2-methoxynaphthalene by acetyl chloride in sulfolane at $150^{\circ} \mathrm{C}^{\mathrm{a}}$

\begin{tabular}{lllll}
\hline Cycle & Conversion $(\%)^{\mathrm{b}}$ & \multicolumn{3}{c}{ Selectivity to different isomers $(\%)^{\mathrm{b}}$} \\
\cline { 3 - 5 } & & 1-acyl & 6-acyl & 8 -acyl \\
\hline 1 & 40 & 23 & 69 & 8 \\
2 & 36 & 21 & 71 & 8 \\
3 & 33 & 27 & 65 & 8 \\
\hline
\end{tabular}

${ }^{\text {a }}$ Substrate:acylating agent 1:2.

${ }^{\mathrm{b}}$ After $6 \mathrm{~h}$ reaction time. 
Excellent regenerability of H-mordenite catalyst was observed, as shown in Table 3. Although there was slight loss of activity (due to loss of aluminum), the selectivity towards different acylated isomers was almost unchanged up to three catalytic cycles.

\section{Conclusions}

H-mordenite showed very good activity for liquid phase acylation of 2-methoxynaphthalene. The final product selectivity was found to be dependent more on the nature of the acylating agents and the reaction temperature than on the type of the zeolite structure. With acetyl chloride as the acylating agent, rearrangement of the primary product, 1-acyl isomer to 6- or 8-acyl isomer, was predominant. The presence of extra-framework aluminum in the catalyst leads to a very high selectivity towards desired 6-acyl isomer, possibly through formation of strong acid centers with acetyl chloride. H-mordenite catalyst also showed excellent regenerability for repeated use.

Comparison with other zeolite catalysts shows that at moderate temperature $\mathrm{H}$-beta has better selectivity for the 6-acyl isomer than the HY and H-mordenite. However, at higher temperatures, yields of the 6-acyl isomer were found to be similar with all three catalysts due to faster isomerization of the 1-acyl isomer to 6-acyl isomer.

\section{Acknowledgements}

The financial support and a post-doctoral research fellowship (DD) from the National Science Council,
Taiwan, are gratefully acknowledged. The authors are also thankful to Tosoh Chemicals, Japan, for a free sample of H-mordenite catalyst and to Dr. Hong-Ping Lin for recording the ${ }^{27} \mathrm{Al}$ MAS NMR spectra.

\section{References}

[1] G.A. Olah, Friedel-Crafts Chemistry, Wiley, New York, 1973.

[2] B. Chiche, A. Finiels, C. Gauthier, P. Geneste, J. Org. Chem. 51 (1989) 2128.

[3] R. Fang, G. Harvey, H.W. Kouwenhoven, R. Prins, Appl. Catal. A: General 130 (1995) 67.

[4] Y.V. Subba Rao, S.J. Kulkarni, M. Subrahmanyam, A.V. Rama Rao, Appl. Catal. A: General 133 (1995) L1.

[5] A. Corma, M.J. Climent, H. García, J. Primo, Appl. Catal. 109 (1996) 177

[6] K. Gaare, D. Akporiaye, J. Mol. Catal. 49 (1989) 109.

[7] Y. Ma, Q.L. Wang, W. Jiang, B. Zuo, Appl. Catal. A: General 165 (1997) 199.

[8] K. Smith, Z. Zhenhua, P.K.G. Hodgson, J. Mol. Catal. A. 134 (1998) 121

[9] B.M. Choudary, M. Sateesh, M.L. Kantam, K.V.R. Prasad, Appl. Catal. A: General 171 (1998) 155

[10] G. Harvey, G. Mäder, Collect. Czech. Comm. 57 (1992) 862.

[11] E.A. Gunnewegh, S.S. Gopie, H. van Bekkum, J. Mol. Catal. A. 106 (1996) 151.

[12] P.J. Harrington, E. Lodewijk, Org. Proc. Res. Dev. 1 (1997) 72.

[13] P.H. Gore, in: G.A. Olah (Ed.), Friedel-Crafts and Related Reactions, Vol. 3, Wiley, New York, 1964, pp. 72.

[14] J. Al-Kabi, J.A. Farooqi, P.H. Gore, A.M.G. Nassar, E.F. Saad, E.L. Short, D.N. Waters, J. Chem. Soc., Perkin Trans. II, 1998, 943.

[15] G.C. Bond, Heterogeneous Catalysis, Principles and Applications, Oxford Science Publications, Oxford, 1987, p. 52 . 